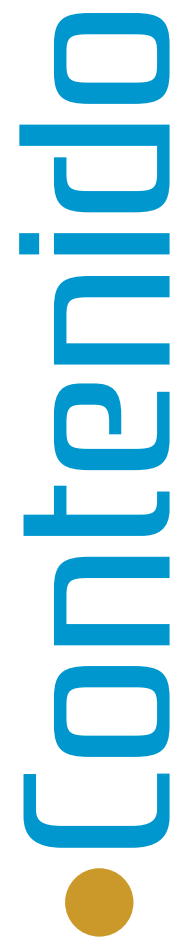

\title{
Editorial
}

Educación en tiempos de pandemia: estrategias

para la mejora de los procesos educativos en el contexto universitario.

Jensy Campos Céspedes

\section{Editorial Científico}

Volver a la Pedagogía

Daniel Prieto Castillo

Cambios educativos en tiempos de pandemia .

Antonio Bartolomé-Pina

Educación en tiempos de pandemia: tecnologías digitales en la mejora

de los procesos educativos

Jesús Salinas Ibáñez

Tiempo de transformación educativa.

Albert Sangrà

Transformación educativa mediada con tecnología digital: oportunidad única de cara a la "nueva normalidad"

Álvaro H. Galvis

El duelo educativo y el rediseño como desafío de superación

Leonel Ángel Carlini López

\section{EnSAYOS}

Educación Superior en Tiempos de COVID-19: oportunidades y retos de la educación

a distancia ....

Ana Cristina Umaña Mata

Tiempos de pandemia y enseñanza universitaria: recrear las propuestas metodológicas entre el hogar y las plataformas de medios conectivos Ana Griselda Díaz 
La pandemia COVID 19 en situación de determinismo y libertad:

implicaciones para el sujeto, el individuo y la persona.

Oscar Eligio Villanueva Gutiérrez y Leticia Isabel Lopez Lopez

\section{SISTEMATIZACIONES}

Gestión académica y administrativa de la carrera Ingeniería Agronómica de la UNED

y los procesos de investigación y extensión asociados en tiempos de COVID-19

Laura Sánchez Calvo, Alfonso Rey Corrales, Priscilla Morales Córdoba, Adriana Mora Jiménez, Marco Córdoba Cubillo,

Karla Montero Jara, Wagner Peña Cordero, Andrés Zúñiga, Karla Mora Rivera, Patricia Oreamuno Fonseca,

Adrián José Bonilla Mora, Catalina Vargas-Meneses

La experiencia de una conferencia virtual para formación profesional

en Educación Matemática de primaria en Geometría

Marianela Zumbado-Castro

El trabajo experimental en la enseñanza de la Física en tiempos de pandemia mediante el uso de la aplicación II Ley de Newton en la UNED de Costa Rica. .

Eduardo Arias Navarro y Carlos Arguedas-Matarrita

El problema social de la educación virtual universitaria en tiempos de pandemia, Perú

Jesús Wiliam Huanca Arohuanca, Felipe Supo Condori, Reynaldo Sucari León y Luis Alberto Supo Quispe

Reinventando la práctica docente en la Universidad Estatal a Distancia de Costa Rica: una oportunidad de crecimiento profesional en tiempos de COVID-19

Zarelly Sibaja Trejos, Nohemí Hernández Herrera y Rafael Ángel Granados Portugués

\section{ARTÍCULOS}

Percepciones del estudiantado argentino de nivel superior acerca del compromiso, clima del aula virtual y tendencias a futuro: entre posibilidades y limitaciones en tiempos de pandemia.

Daiana-Yamila Rigo

Desafíos y oportunidades en tiempos del COVID-19: contexto pedagógico desde la Universidad Nacional y la Universidad Estatal a Distancia

Priscilla Carranza-Marchena y Geraldine Zamora-Sánchez

La docencia en el nivel de posgrado en el contexto de virtualización de emergencia. Aprendizajes y desafíos para el futuro en la experiencia de la Universidad Nacional de Tres de Febrero

Marisa Álvarez, Norberto Fernández Lamarra, Pablo Daniel García, María Eugenia Grandoli, y Cristian Perez Centeno

BOT0210, una herramienta de apoyo a la docencia. Experiencia de la Cátedra Tecnologías de la Información de la UNED, Costa Rica

Luis Monge Mata y Carolina Ávalos Dávila

Estrategias de acompañamiento virtual en un grupo de teatro inclusivo durante tiempos de distanciamiento social.

Alicia Sandoval Poveda y Linda Madriz Bermúdez

La bitácora como estrategia didáctica en el curso de Introducción a la Pedagogía de la Universidad de Costa Rica durante la crisis del COVID-19.

Juan Carlos Naranjo Segura

La gestión curricular en centros educativos públicos de Costa Rica durante la pandemia del virus de la COVID-19

Satya Rosabal Vitoria y Yors Guillermo Solis Vargas

Estrategias didácticas empleadas desde la presencialidad remota en la División de Educación para el Trabajo de la Universidad Nacional en tiempos de pandemia...................... Ana Lidieh Montes Rodríguez, Víctor Villalobos y Warner Ruiz Chaves 


\section{Directora - Editora}

Jensy Campos Céspedes

Universidad Estatal a Distancia, Costa Rica.

\section{Consejo Editorial \\ Adrián Solano Castro \\ Universidad Estatal a Distancia, Costa Rica. \\ Andrea Rojas Vargas \\ Universidad Estatal a Distancia, Costa Rica. \\ Rosberly Rojas Campos \\ Universidad Estatal a Distancia, Costa Rica. \\ Yarith Rivera Sánchez \\ Universidad Estatal a Distancia, Costa Rica. \\ Viviana Berrocal Carvajal \\ Universidad Estatal a Distancia, Costa Rica.}

\section{Consejo Científico Nacional \\ Carlos Vargas Loáiciga \\ COLYPRO, Costa Rica \\ Francisco Romero Estrada \\ Universidad de Costa Rica, Costa Rica. \\ Gilberto Alfaro Varela \\ Universidad de Costa Rica, Costa Rica. \\ Julieta Solórzano Salas \\ Universidad de Costa Rica, Costa Rica. \\ Luis Ángel López Ruiz \\ Universidad de Costa Rica, Costa Rica. \\ Mónica Arias Monge \\ Universidad de Costa Rica, Costa Rica \\ Rafael Jiménez Corrales \\ Universidad Nacional, Costa Rica. \\ Rocío Ramírez González \\ COLYPRO, Costa Rica.}

\section{Consejo Científico Internacional}

Antonio Bartolomé Pina

Universitat de Barcelona. España.

Antonio Nolberto De Oliveira Xavier

Universidade Estadual de Santa Cruz, Brasil.

Bartolomé Chinchilla Chinchilla

Universidad Pedagógica Nacional Francisco Morazán, Honduras.

\section{COMITÉ EDITORIAL}

\section{Bernardo Trejos Murillo}

I-Shou University, Provincia de Taiwán.

Claudia Martínez Olhagaray

Universidad de la República, Uruguay.

Dulce Fátima Camacho Gutiérrez

Instituto Tecnológico de Monterrey. México.

Edith Soria Valencia

Pontificia Universidad Católica, Perú.

Elena Barberà Gregori

Universitat Oberta de Catalunya, España.

Emiliano Diez Villoria

Universidad de Salamanca, España.

Facundo Corvalán

Universidad Nacional de Rosario, Argentina.

Gina Garcés Ruíz

Universidad Especializada de las Américas, Panamá.

Karl Steffens

Universidad de Colonia, Alemania.

Leonel Carlini López

Universidad Tecnológica Nacional, Argentina.

Luz Díaz Subieta

Universidad Católica Luis Amigó, Colombia.

Manuel Rosales Almendra

Universidad de Guadalajara, México.

Norberto Fernández Lamarra

Universidad Nacional de Tres de Febrero, Argentina.

Paloma Antón Ares

Universidad Complutense de Madrid, España.

Raúl Vela Sosa

Universidad Autónoma de Yucatán, México.

Rogelio Ruiz Ríos

Universidad Autónoma de Baja California, México.

Sara Fiallos Varela

Universidad Nacional Autónoma de Honduras, Honduras.

Sixto Moya Herrera Investigación y Estudios Educativos y Sindicales de la Actualidad, México.

Teresa Ribeiro Pessoa Universidade de Coimbra, Portugal

Víctor Torres Covarrubias

Universidad Autónoma de Nayarit, México.

Yolanda Camacho González

Universidad Autónoma de Nayarit, México.

Innovaciones Educativas es una publicación académica de la Universidad Estatal a Distancia (UNED) que se realiza en los meses de junio y diciembre. Las responsabilidad compete a los autores. Es una revista de acceso abierto, y se ampara bajo una Licencia Creative Commons Atribución-NoComercial 4.0 Internacional. En tal sentido, se autoriza la reproducción total o parcial de los artículos siempre y cuando se reconozca la autoría de la obra y se cite correctamente la fuente; por tal motivo, debe añadirse además el enlace URL de la revista. Consulte las normas editoriales en el portal de la revista. Servicios de información:

\section{CONTACTO}

Universidad Estatal a Distancia, Escuela de Ciencias de la Educación, Centro de Investigaciones en Educación (CINED). Sede Central, Edificio C, Piso 2. De la Rotonda La Betania 500m al este, Carretera a Sabanilla, Mercedes de Montes de Oca. Ap. postal 474-2050. San José, Costa Rica. • Teléfono: (506) 2527-2427 • Correo electrónico: innoveducativas@uned.ac.cr. Para envíos consultar las normas editoriales en la siguiente dirección electrónica: https://revistas.uned.ac.cr/index.php/innovaciones.

Attribution-NonCommercial CC BY-NC - Esta obra está bajo una Licencia Creative Commons Atribución-NoComercial 4.0 Internacional. (BY) You must give appropriate credit, provide a link to the license, and indicate if changes were made. You may do so in any reasonable manner, but not in any way that suggests the licensor endorses you or your use. (NC) NonCommercial — You may not use the material for commercial purposes. 


\section{Editorial}

\section{Educación en tiempos de pandemia: estrategias para la mejora de los procesos educativos en el contexto universitario}

En un contexto inédito como lo es la pandemia, con repercusiones a nivel planetario, en todos los ámbitos de la vida humana, desde la Revista Innovaciones Educativas les ofrecemos un número especial, por medio del cual nos acercamos a las realidades experimentadas en diferentes escenarios de la educación durante la pandemia por el virus responsable de la COVID-19.

En primer lugar, obliga el entero agradecimiento por un lado a todas las personas autoras que nos dignan con sus contribuciones en este número especial y, por otra parte, a ustedes, fieles lectores, para quienes nuestra revista tiene razón de existir.

En este número especial hemos ampliado el tipo de contribuciones; por lo tanto, se ofrece una selección de trabajos académicos bajo el formato de editorial científico, artículo, ensayo y sistematización de experiencias, los cuales someramente describiremos en las siguientes líneas.

Nuestro primer trabajo lo constituye el aporte de Daniel Prieto Castillo, quien con un itinerario superior a tres décadas de práctica de mediación pedagógica en espacios educativos formales y no formales, con énfasis en actividades de promoción y acompañamiento del aprendizaje en sistemas presenciales y a distancia, nos plantea que en tiempos de pandemia, aun cuando podamos superar lo que significa la apropiación de recursos tecnológicos, el reto más importante se centra en trascender las carencias pedagógicas.

Seguidamente, Antonio Bartolomé Piña en su ensayo titulado "Cambios educativos en tiempos de pandemia", nos alerta sobre las brechas que supone la coexistencia de una sociedad en red y una universidad tradicional, así como los cambios que se tornan insoslayables para la universidad.

Por su parte, Jesús Salinas Ibáñez nos aporta un documento en el cual -con gran claridad-expone algunas debilidades del sistema educativo que se han manifestado en el contexto de la pandemia y enfatiza sobre la necesidad de orientar esfuerzos hacia un rediseño radical de la enseñanza, así como una reinvención de la carrera docente en la cual, entre otros aspectos, se incorpore el desarrollo de la competencia digital.

Albert Sangrà Morer expone como el carácter imprevisto de la pandemia obligó a los sistemas edcuativos del mundo a recurrir a soluciones virtuales y hace énfasis en la necesidad de aprovechar la coyuntura para generar modelos educativos híbridos que permitan transitar entre momentos presenciales y momentos no presenciales del proceso educativo. 
En ese mismo sentido, Álvaro H. Galvis nos ofrece un escrito en donde alude a la vertiginosidad del cambio para la educación, esto ha implicado la utilización de Tecnologías Digitales (TD) para apoyar procesos educativos y la gestión durante la pandemia. A la vez que plantea la necesidad de aprovechar la coyuntura para propiciar transformación educativa mediada con TD, la cual involucre las dimensiones educativa, tecnológica, organizacional y cultural.

En otro orden de ideas, Leonel Ángel Carlini López nos expone la manera en que la pérdida de los formatos educativos tradicionales impulsó el rediseño de la tríada didáctica debido a la virtualidad y cómo esa ruptura configuró al aula virtual como una legalidad democratizadora, en donde el personal docente se enfrenta al desafío de recuperar la pulsión epistemofílica.

En cuanto a los aportes del formato de ensayo, Ana Cristina Umaña Mata realiza una presentación general de algunas de las principales oportunidades y retos del modelo educativo a distancia; ofrece este modelo como una de las opciones educativas con mayores posibilidades de desarrollo a nivel latinoamericano, ante las condiciones derivadas de la crisis sanitaria.

Sobre ese mismo eje temático, Ana Griselda Díaz aporta una reflexión sobre la enseñanza universitaria y la necesidad de incluir nuevos contenidos y formas de enseñanza con cara a escenarios profesionales cambiantes e inciertos.

En un orden más filosófico, Oscar Eligio Villanueva Gutiérrez y Leticia Isabel López López proponen una reflexión acerca del determinismo y la libertad como marco para pensar la pandemia producida por la COVID-19 en las tres condiciones del ser humano: sujeto, persona e individuo. Desde la perspectiva de estas personas autoras es posible mejorar el sistema educativo en tiempos de reformas educativas y de pandemia, solamente si el sistema determinista admite que necesita la profesionalidad del docente para trabajar la integración curricular y pedagógica, las Tecnologías de la Información y la Comunicación (TIC) y las prácticas académicas colectivas.

En cuanto a la sistematización de experiencias, Laura Sánchez Calvo, Alfonso Rey Corrales, Priscilla Morales Córdoba, Adriana Mora Jiménez, Marco Córdoba Cubillo, Karla Montero Jara, Wagner Peña Cordero, Andrés Zúñiga Orozco, Karla Mora Rivera, Patricia Oreamuno Fonseca, Adrián José Bonilla Mora y Catalina VargasMeneses comparten lo que, desde la carrera de Ingeniería Agronómica de la UNED, se ejecutó durante la emergencia sanitaria, como parte de los procesos de gestión académica y administrativa, así como la articulación con los procesos de investigación y extensión asociados.

En el mismo contexto institucional, Marianela Zumbado-Castro comparte la experiencia de una conferencia virtual para formación profesional en Educación Matemática de primaria en Geometría; mientras que Eduardo Arias Navarro y Carlos Arguedas-Matarrita socializan el trabajo experimental en la enseñanza de la Física realizado mediante el uso de la aplicación II Ley de Newton.

Seguidamente, Jesús Wiliam Huanca Arohuanca, Felipe Supo Condori, Reynaldo Sucari León y Luis Alberto Supo Quispe presentan los resultados de un estudio en cuatro universidades peruanas, en el cual señalan las dificultades que representan las debilidades asociadas con las habilidades docentes y la falta de acceso a internet por parte del estudiantado para la implementación de la educación virtual.

Zarelly Sibaja Trejos, Nohemí Hernández Herrera y Rafael Ángel Granados Portugués comparten el trabajo desplegado por la Escuela de Ciencias de la Educación de la Universidad Estatal a Distancia de Costa Rica, para ofrecer continuación a las Prácticas Docentes y Profesionales de esta unidad académica, durante el periodo de aislamiento social.

Bajo el formato de artículo, encontramos el trabajo de Daiana-Yamila Rigo, en el cual nos da cuenta de una investigación desarrollada en tres instituciones educativas de Argentina, cuyo objetivo se centró en comprender 
las perspectivas construidas por los estudiantes remotos sobre compromiso académico asumido y clima del aula virtual en contexto de pandemia en el nivel superior de educación.

Seguidamente, se halla el manuscrito de Priscilla Carranza-Marchena y Geraldine Zamora-Sánchez, quienes nos invitan a reflexionar acerca de los desafíos y las oportunidades de mejora que el profesorado enfrentó a causa de la pandemia en dos universidades públicas de Costa Rica.

Con algunos resultados de una investigación sobre docencia en el nivel de posgrado, Marisa Álvarez, Norberto Fernández Lamarra, Pablo Daniel García, María Eugenia Grandoli y Cristian Pérez Centeno, nos brindan el artículo titulado "La docencia en el nivel de posgrado en el contexto de virtualización de emergencia. Aprendizajes y desafíos para el futuro en la experiencia de la Universidad Nacional de Tres de Febrero". En este documento, nos informan sobre algunas particularidades del giro rotundo de la presencialidad tradicional a la remota experimentada en el nivel de posgrado de su universidad durante la pandemia.

Luis Monge Mata y Carolina Ávalos Dávila, por su parte, nos comparten resultados de un estudio que realizaron, el cual se vincula con el uso de la herramienta BOT0210 para apoyar la docencia en una cátedra dentro de la Universidad Estatal a Distancia de Costa Rica.

En otro orden de temas, pero también muy relevante, Alicia Sandoval y Linda Madriz Bermúdez nos ofrecen un interesante artículo sobre el desarrollo de estrategias de acompañamiento virtual implementadas en un grupo de teatro inclusivo, durante tiempos de distanciamiento social. Las autoras nos informan cómo, en la virtualidad, se han desarrollado espacios de acompañamiento emocional para afrontar la ansiedad que experimentan las personas en situación de discapacidad durante el proceso de distanciamiento social.

Con el fin de seguir con las estrategias didácticas, Juan Carlos Naranjo Segura aporta un diagnóstico relacionado con el uso de la bitácora, como estrategia didáctica durante la emergencia sanitaria en una asignatura de la Escuela de Formación Docente de la Universidad de Costa Rica.

Relacionado con la gestión educativa, Satya Rosabal Vitoria y Yors Guillermo Solís Vargas quienes analizan el papel de la gestión curricular en centros educativos públicos costarricenses en tiempos de la pandemia provocada por el virus de la COVID-19. Según las personas autoras, el personal directivo de las organizaciones educativas requiere las habilidades necesarias para afrontar los desafíos educativos derivados de la crisis sanitaria y son aquellas vinculadas con el ámbito de la gestión del currículo.

Cierran este número especial, Ana Lidieth Montes Rodríguez, Víctor Villalobos Benavides y Warner Ruiz Chaves nos ofrecen un manuscrito con los resultados de un estudio relacionado con las estrategias didácticas utilizadas en una unidad académica de la Universidad Nacional (UNA) de Costa Rica para implementar la presencialidad remota como respuesta a los cambios en los procesos educativos que se dieron durante el periodo de confinamiento a raíz de la pandemia.

Con este número especial esperamos contribuir al proceso de búsqueda de soluciones, que en las distintas latitudes realizan profesionales de la educación de todos los niveles educativos para responder pertinentemente a los desafíos insoslayables que la pandemia le plantea a los sistemas educativos y los cambios que supondrá la nueva normalidad que se establecerá después de la emergencia sanitaria. 